\title{
Competencias y curriculum en educación de adultos: hábitos docentes en Asturias
}

\author{
Susana Molina-Martín, Beatriz Sierra- \\ Arizmendiarrieta \& Marta Soledad García- \\ Rodríguez
}

\begin{abstract}
Resumen
En España la introducción de las competencias en educación se produjo en 2006, adquiriendo especial importancia las competencias clave, entendidas como una combinación de conocimientos, capacidades y actitudes adecuadas al contexto e identificándolas como aquellas que todas las personas precisan para su realización y desarrollo personal, así como para la ciudadanía activa, la inclusión social y el empleo. Se proponen tres perfiles docentes en relación a las competencias: enseñanza tradicional, modelo en transición y trabajo por competencias. El objetivo de la presente investigación es ubicar al profesorado que trabaja en los Centros de Educación de Personas Adultas en dichos perfiles docentes. La muestra está formada por 85 docentes de educación de adultos de los que el $49,4 \%$ son hombres y el $50,6 \%$ mujeres. Para recoger la información se elaboró un cuestionario que consta de una primera parte, para obtener datos del carácter sociológico de la muestra, y una segunda, compuesta por cincuenta ítems, con una escala tipo Likert de cuatro niveles de respuesta. Los resultados indican que el $31,76 \%$ del profesorado trabaja para el desarrollo de competencias mientras que el $68,24 \%$ restante se sitúa en un modelo en transición hacia el trabajo por competencias.
\end{abstract}

Palabras clave:

competencias; educación de adultos; evaluación; formación del profesorado; metodología. 


\title{
Competences and curriculum in adult education: teaching habits in Asturias
}

\begin{abstract}
In Spain, the introduction of competences in education occurred in 2006, acquiring special importance the key competences, understood as a combination of knowledge, skills and attitudes appropriate to the context and identifying them as those that all people need for their realization and personal development, as well as for active citizenship, social inclusion and employment. Three teaching profiles are proposed in relation to the competences: traditional education, model in transition and work by competences. The objective of the present investigation is to locate the teaching staff that works in Adult Education Centers in these teaching profiles. The sample consists of 85 teachers of adult education of which $49.4 \%$ are men and $50.6 \%$ women. To collect the information, a questionnaire consisting of a first part, to collect data on the sociological nature of the sample, and a second, consisting of fifty items, with a Likert scale of four response levels, was built. The results indicate that $31.76 \%$ of the faculty work for the development of competences while the remaining $68.24 \%$ is placed in a model in transition to work by competences.
\end{abstract}

Key words: competencies; adult education; methodology; assessment; teacher training.

\section{Competências e currículo na educação de adultos: hábitos de ensino nas Astúrias}

Resumo: Em Espanha, a introdução de competências na educação ocorreu em 2006, adquirindo especial importância as competências-chave, entendidas como uma combinação de conhecimentos, habilidades e atitudes adequadas ao contexto e identificando-as como aquelas que todas as pessoas necessitam para a sua realização e desenvolvimento pessoal, bem como para uma cidadania ativa, inclusão social e emprego. São propostos três perfis de ensino em relação às competências: ensino tradicional, modelo em transição e trabalho por competências. O objetivo desta pesquisa é colocar o corpo docente dos Centros de Educação de Adultos nesses perfis de ensino. A amostra é constituída por 85 professores de educação de adultos, dos quais 49,4\% são homens e 50,6\% mulheres. Para coletar as informações, foi elaborado um questionário composto por uma primeira parte, para coletar dados sobre a natureza sociológica da amostra, e um segundo, composto por cinquenta itens, com uma escala Likert de quatro níveis de resposta. Os resultados indicam que 31,76\% dos docentes trabalham para o desenvolvimento de competências, enquanto os $68,24 \%$ restantes são colocados em um modelo em transição para o trabalho por competências.

Palavras-chave: competências; educação de adultos; avaliação; formação de professores; metodologia.

\section{Compétences et programme d'études en éducation des adultes: habitudes d'enseignement dans les Asturies.}

Résumé : En Espagne, l'introduction des compétences en éducation s'est produite en 2006 et dès lors, les compétences clé ont acquis une spéciale relevance. Celles-ci sont interprétées comme une intégration de connaissances, de capacités et d'attitudes adaptées au contexte, et comme étant nécessaires pour l'accomplissement et le développement personnel, la citoyenneté active, l'inclusion sociale et l'emploi. On y propose trois profils des enseignants par rapport aux compétences: un enseignement traditionnel, un modèle en transition et un travail par compétences. L'objectif de cette recherche, c'est de situer les enseignants des Centres d'Éducation pour Adultes par rapport à ces profils. L'échantillon est composé par 85 enseignants, dont 49,4\% sont des hommes et 50,6\% des femmes. Pour recueillir les informations, on a créé un questionnaire comprenant une première partie, destinée à collecter des données sur la nature sociologique de l'échantillon, et un second, composé de cinquante items, sur une échelle de type Likert de quatre niveaux de réponse. Les résultats signalent que 31,76\% des enseignants travaillent pour le développement des compétences, tandis que les $68,24 \%$ restants se situent dans un modèle en transition ver le travail par compétences.

Mots clés: compétences; éducation des adultes; méthodologie; évaluation; formation des enseignants. 


\section{Introducción y estado de la cuestión}

La educación de adultos es un saber educativo específico en el conjunto de las Ciencias de la Educación, de modo que alude a un conjunto de prácticas diferenciadas, con una idiosincrasia propia (Medina Fernández, 2000; Sarrasate Capdevila, Pérez de Guzmán Puya, 2005). Hay muchos rasgos diferenciadores que pueden abordarse desde una perspectiva interdisciplinar, aunque vamos a destacar sólo algunos, que nos parecen especialmente relevantes desde el punto de vista psicológico y pedagógico, entre los que cita Medina Fernández (2000), y que distinguen claramente este tipo de educación de la que se produce en el ámbito escolar: motivación asegurada, pues habitualmente los adultos participan de forma voluntaria; experiencia como fuente y contraste de su aprendizaje; necesidad de un aprendizaje como solución de problemas de su vida cotidiana, familiar, social y laboral; tiempo de dedicación parcial, debido a sus diferentes ocupaciones; autonomía en la toma de decisiones y capacidad para asumirlas. Junto con ello, en la educación de adultos nos encontramos frecuentemente con la necesidad de: mejorar la autoestima, superar miedos, aplicar los aprendizajes a la vida diaria y tener la oportunidad de demostrar y expresar lo aprendido. Por eso, en este tipo de educación, tiene especial relevancia la participación y el diálogo, tanto para facilitar el aprendizaje, como para mejorar la autoestima e incrementar y mantener la motivación.

Se hace evidente que una educación con tales características necesita no sólo metodologías y actividades adaptadas a estas peculiaridades, sino un profesorado que sepa entenderlas y ajustarse a ellas. Además, en un momento de cambio curricular para todas las etapas educativas, es preciso resaltar la Recomendación 2006/962/ CE, del Parlamento Europeo y del Consejo, de 18 de diciembre de 2006, sobre las competencias clave para el aprendizaje permanente. En ella se insta a los Estados miembros a desarrollar la oferta de las competencias clave, entendiendo estas como una combinación de conocimientos, capacidades y actitudes adecuadas al contexto e identificándolas como aquellas que todas las personas precisan para su realización y desarrollo personal, así como para la ciudadanía activa, la inclusión social y el empleo.

La enseñanza basada en competencias parece algo nuevo en el panorama educativo y, desde su implantación, ha provocado resistencias, confusión y clamor por una mayor formación docente para hacer frente a los nuevos requerimientos curriculares. Desde el punto de vista formal, se ha ido conformando una nueva terminología que inquieta al profesorado. No obstante, si vamos al fondo de la cuestión nos encontramos con una antigua demanda que continúa de actualidad: aprender de forma significativa y a partir de la experiencia, de los problemas y contextos cotidianos. Ausubel y, aún más lejos, Rousseau ya preconizaban la necesidad de tales cambios (Sierra-Arizmendiarrieta, Pérez-Ferra, 2015). 


\section{Cambio metodológico y evaluación: claves de una transformación curricular}

En España la introducción de las competencias en el curriculum se produjo en 2006 y se planteó de forma paulatina. Cualquier reforma educativa encuentra obstáculos, de carácter individual y social, para su implantación. Esta última no podía ser menos y ha encontrado barreras semejantes. Una vez completada la fase de incorporación plena de las competencias en el currículo, una investigación sobre la percepción de los maestros de Primaria de Asturias sobre el cambio prescrito, puso de manifiesto que el profesorado de esta etapa no parecía estar plenamente convencido de sus ventajas, dejando entrever poca credibilidad en las competencias básicas, lo que podría mermar el deseo de cambio (Méndez-Giménez, Sierra-Arizmendiarrieta, MañanaRodríguez, 2013). Por otra parte, en el mismo año se publica un estudio cualitativo de casos múltiples en tres centros educativos públicos de enseñanza Secundaria de tres comunidades autónomas españolas (Madrid, Castilla-La Mancha, y Castilla y León), poniendo de manifiesto la gran distancia entre las pretensiones normativas del cambio educativo, para la formación en competencias, y lo sucedido realmente en las aulas (Monarca, Rappoport, 2013). El propio profesorado entrevistado destaca, entre otras, como causas de este fracaso: la falta de formación y asesoramiento, la falta de oportunidad de participación, y un exceso de vigilancia normativa sin repercusión en los procesos ocasionando que se descuiden a favor de la burocratización.

No hemos encontrado investigaciones sobre el desarrollo de las competencias clave en educación de adultos, o la percepción del profesorado implicado en ella. Por este motivo, nos planteamos realizar una primera aproximación analizando si los hábitos docentes del profesorado de educación de adultos en Asturias reflejan los cambios que la formación en competencias exige. Intentando reducir dichos cambios a lo esencial, este cambio de enfoque exige una transformación metodológica y, por ende, evaluativa.

Algunos autores, centrados en la enseñanza superior, han puesto de manifiesto que metodología y evaluación son las claves de la transformación curricular exigida (Mateo y Vlachopoulos, 2013). Pensamos que, aunque la formación en competencias tiene peculiaridades para las diferentes etapas, en lo relativo a estos dos cambios puede haber un acuerdo común. Otra cosa será, el tipo de actividades o la exigencia en los aspectos de las competencias a evaluar. Por ello, vamos a señalar, con carácter general, los avances que deberían darse en estos dos aspectos.

La formación en competencias, para cualquier alumnado, exige al menos dos grandes cambios metodológicos: en el aula y fuera del aula (Sierra-Arizmendiarrieta, Méndez-Giménez, Mañana-Rodríguez, 2013). La metodología de aula pasa por el cambio en el tipo de actividades y por una mayor interrelación entre los contenidos. Las competencias se ejercitan y desarrollan resolviendo problemas en diversas situaciones, lo 
que hace imposible separar los contenidos. Estos han de conectarse, realizando actividades interdisciplinares que imiten lo más posible las situaciones reales en las que se encuentra el alumnado en su vida diaria. Así, las actividades no han de plantearse para demostrar el dominio de unos contenidos con los que quizá no se sabe qué hacer, sino para llegar a saber desenvolverse, de forma eficaz, en distintas situaciones. Esto implica, por ejemplo, la exigencia de una mayor independencia del docente respecto al libro de texto, como un recurso de apoyo constante. Sáiz Serrano (2011), tras un análisis de manuales de Historia de $1^{\circ}$ y $2^{\circ}$ de ESO, concluye que, tras la implantación de las competencias básicas, estos manuales no facilitan su formación, y continúan con actividades de bajo nivel cognitivo y eminentemente reproductivas. Además de resolver situaciones, algunos estudiantes universitarios perciben más útiles las actividades que implican discusión e interacción entre ellos (Rubio Hurtado, Ruiz Bueno, Martínez-Olmo, 2016).

Un posible obstáculo a un planteamiento más exigente e interdisciplinar de actividades sería la imposibilidad de que un mismo docente dominara todos los contenidos implicados en ellas, por lo que enlazamos con la necesidad del segundo cambio metodológico, ya fuera del aula, y sustrato del primero: un trabajo cooperativo interdisciplinar con otros docentes (Bolarín Martínez, Moreno Yus, Porto Currás, 2013; Cárdenas-Rodríguez, Terrón-Caro, Monreal-Gimeno, 2015; Karrera, Zulaika, Aldaz, 2014; Lavega, Sáez De Orcáriz, Lasierra, Salas, 2013; Palomares Montero, ChisvertTarazona, 2016), de forma que se compartan experiencias y saberes y se puedan planificar actividades más enriquecedoras y motivadoras para los estudiantes. Ligado a este planteamiento, y entre las metodologías innovadoras que más pueden favorecer el desarrollo de diversas competencias, se encuentran el aprendizaje cooperativo (Silva, Lopes, Dominguez, Payan-Carreira, Morais, Nascimento, Morais, 2016), el aprendizaje basado en la resolución de problemas (Ferreira, Henriques-Coelho, 2016; Hidalgo, de la Blanca, Risueño, 2011) y el coaching (Sánchez Mirón, Boronat Mundina, 2014), entre otros.

En lo que respecta a la evaluación, hay que decir que no se pueden evaluar directamente las competencias, pues si éstas han de demostrarse en la resolución de problemas en diferentes contextos, la evaluación tendría que quedar emplazada al futuro. En este sentido y refiriéndose a sistemas de reconocimiento y acreditación de la experiencia, Medina Fernández y Sanz Fernández (2009) afirman que no se evalúan las competencias, sino las capacidades, a través de diferentes evidencias. Estas pueden ser la ejecución de una acción profesional, sea real o simulada, o cualquier producción oral y escrita relacionada con las anteriores, a modo de un producto (informe, cuestionario, proyecto...). De acuerdo con ellos, podríamos añadir que cada una de dichas evidencias refleja un 'aspecto competencial situado', es decir, la demostración -en un contexto concreto- de una capacidad que revela o indica la posesión de cierta 
competencia, al menos en parte. Pero si se quiere precisar la adquisición y desarrollo competencial, es preciso la determinación de distintos niveles de dominio, definiendo muy bien cada uno, y el establecimiento de indicadores que ayuden a valorar el progreso (Villa, Poblete, 2007).

Villardón (2006) distingue entre "evaluación de competencias" y "evaluación para el desarrollo de competencias". La primera persigue la certificación de unas competencias ya logradas, para su reconocimiento, realizando la función sumativa de la evaluación. La segunda representa el ejercicio de la función formativa, orientada a favorecer y ayudar al logro de las competencias deseadas. Ésta ha de llevarse a cabo durante todo el proceso, pues supone un instrumento de mejora hasta el logro de la acreditación correspondiente.

Una propuesta innovadora para la "evaluación para el desarrollo", como complemento a otros instrumentos, es La Entrevista de Evaluación del Aprendizaje Focalizada en Competencias (Villa, Poblete, 2011). Supone un diálogo centrado sobre una competencia, que induce al estudiante a la reflexión sobre sus puntos fuertes y débiles, de forma que participe activamente de su propia evaluación y ésta sirva como medio para la mejora y mayor desarrollo de la competencia y no exclusivamente para detectar los fallos. Resulta especialmente relevante la inmediatez y el carácter formativo del feedback que se produce. Respecto a la "evaluación de competencias", al final de procesos formativos, resulta interesante el SEBSCO (sistema de evaluación basado en situaciones complejas) (Poblete, Villa, 2011). Se trata del diseño de pruebas para evaluar el nivel de desempeño de competencias genéricas y específicas en una situación profesional simulada y en un contexto que podría -o no- ser real. Aunque los dos instrumentos de evaluación señalados se plantean para la etapa universitaria, pensamos que ambos podrían resultar muy adecuados y motivadores, en la educación de adultos, para evaluar tanto el desarrollo de los procesos como los resultados de cara a una acreditación de competencias.

Teniendo en cuenta cómo se deberían trabajar los diferentes elementos curriculares y organizativos para planificar y desarrollar una enseñanza dirigida al desarrollo de competencias, así como la falta de investigaciones al respecto, especialmente en educación de adultos, se evidencia la necesidad de abordar dicha temática. Por ello, el objetivo de la presente investigación ha sido conocer si el profesorado que trabaja en Centros de Educación de Personas Adultas está desarrollando una enseñanza dirigida al desarrollo de competencias, en la dirección que se ha descrito en los párrafos anteriores. 


\section{Diseño metodológico}

\section{Participantes}

La muestra está formada por ochenta y cinco profesionales de la educación que trabajan en alguno de los once Centros de Educación de Personas Adultas de la red de centros públicos del Principado de Asturias (España). Cabe decir, que según datos del Ministerio de Educación y Formación Profesional (2018) la población de profesores que trabajan en educación de adultos en el Principado de Asturias son ciento cuarenta y seis. Respecto a la muestra, el 94,4\% del profesorado es mayor de 35 años, siendo el $49,4 \%$ hombres y el $50,6 \%$ mujeres. Se trata de una plantilla con una dilatada trayectoria profesional, pues el $80 \%$ de los docentes tiene más de diez años de experiencia y el $43 \%$ está diplomado como profesor.

\section{Instrumento}

Con el objetivo de establecer diferentes perfiles docentes en el desarrollo de competencias se elabora un cuestionario denominado Cuestionario de Perfiles Docentes. Consta de dos partes, una primera dirigida a recoger datos de carácter sociológico de la muestra (centro de trabajo, sexo, edad, años de experiencia y títulos universitarios) y, una segunda, compuesta por cincuenta ítems, con una escala tipo Likert de cuatro niveles de respuesta.

Inicialmente se elaboraron un total de 37 cuestiones, en cada una de ellas se planteaba un elemento curricular y sobre el mismo se planteaban 3 alternativas que tenían que ser evaluadas en una escala tipo Likert, por lo que había un total de 111 ítem A esta primera versión se le calculó el Coeficiente de Validez de Contenido (CVC) que permite valorar el grado de acuerdo de los expertos respecto a cada uno de los diferentes ítems y al instrumento en general, para ello se ha contado con la participación de 5 expertos.

En este trabajo se han seguido las recomendaciones más estrictas y se han considerado los valores del CVC superiores a 0.8. El formato inicial dejó de ser válido y tras varias rondas de valoración por expertos finalmente el cuestionario quedó compuesto finalmente por 50 ítems.

Los ítems del cuestionario tratan de contemplar cómo se plantean los diferentes elementos curriculares y de la organización de los centros necesarios para planificar y desarrollar una enseñanza dirigida al desarrollo de competencias: objetivos, contenidos, materiales y actividades, metodología, evaluación y cuestiones organizativas del centro. 
La valoración de los ítems se realizó en función de los aspectos curriculares y organizativos que se consideraron clave para realizar una formación centrada en el desarrollo de competencias, y que se han recogido en la revisión teórica realizada (ver Tabla 1). En la mayoría de los ítems se ha valorado la respuesta más idónea "siempre" con 4 puntos (ítems 1, 2, 3, 4, 6, 7, 8, 11, 12, 13, 15, 17, 18, 19, 20, 21, 24, 25, 28, $29,30,31,32,33,34,35,36,37,38,39,40,43,45,46,47,48,49,50)$. En otros ítems se han valorado como respuesta más idónea "nunca" con 4 puntos $(9,14,16$, $23,26,27,41,44)$. Hay tres ítems $(5,10,22)$ en los que se valoran de forma positiva las respuestas intermedias "a veces" o "frecuentemente" (4 puntos). Finalmente, el ítem 42 nos permite conocer su opinión sobre la formación docente y no entra a puntuar. Las respuestas mejor valoradas a cada ítem suman 196 puntos.

Tabla 1. Valoración de las respuestas a los ítems del cuestionario

\begin{tabular}{|c|c|c|}
\hline \multicolumn{2}{|c|}{ VALORACIÓN DE LAS RESPUESTAS DE LOS ÍTEMS } \\
\hline ÍTEMS & VALORACIÓN & PUNTOS \\
\hline $1,2,3,4,6,7,8,11,12,13,15,17,18,19,20,21$, & Siempre & 4 \\
$24,25,28,29,30,31,32,33,34,35,36,37,38$, & Frecuentemente & 3 \\
$39,40,43,45,46,47,48,49,50$ & A veces & 2 \\
\hline $9,14,16,23,26,27,41,44$ & Nunca & 1 \\
\hline & Nunca & 4 \\
\hline $5,10,22$ & A veces & 3 \\
\hline 42 & Frecuentemente & 2 \\
\hline & Siempre & \\
\hline
\end{tabular}

Fuente: Elaboración propia

\section{Procedimiento}

Una vez diseñado el cuestionario se realizó una aplicación piloto en un Centro de Educación de Personas Adultas, seleccionado mediante muestreo intencional, por facilidad de acceso y actitud colaboradora de su comunidad educativa. A partir de esta aplicación piloto, se identificaron y modificaron algunos aspectos formales susceptibles de mejora.

Una vez dado por definitivo el cuestionario, se contactó con los equipos directivos de los centros para informarles del objetivo del estudio mediante encuesta. Una vez confirmada su participación, fueron informados, antes de contestar al cuestionario, de su carácter voluntario, anónimo y confidencial. Seguidamente, se administró a través de Google y se les dio un plazo de entrega. Los cuestionarios se aplicaron en el curso académico 2017-2018. 


\section{Análisis de datos}

Para el análisis de datos se ha utilizado el Statistical Package for Social Sciences (SPSS), versión 24.0 para Windows (IBM Corp., 2016). El análisis de fiabilidad de la escala se ha calculado utilizando el alfa de Cronbach, para el resto de los análisis se ha utilizado estadística descriptiva, considerando sumatorios, frecuencias y porcentajes.

\section{Resultados}

A partir de los datos obtenidos de la aplicación del cuestionario, se llevan a cabo los siguientes análisis. En primer lugar, a partir de los datos obtenidos se trata de identificar si el profesorado que trabaja en educación de adultos trabaja por competencias. Seguidamente analizaremos a este último grupo de profesores, es decir, al que afirma trabajar por competencias para conocer sus puntos fuertes y débiles.

\section{Perfiles docentes}

Para identificar si el profesorado que trabaja en educación de adultos trabaja por competencias, se realizará la suma de las puntuaciones obtenidas por cada profesor en el total de ítems (menos el 42), en función de la puntuación otorgada según la respuesta (ver Tabla 1). Según la puntuación obtenida se sitúa a cada profesor (Tabla 2): el profesorado que obtiene una puntuación menor de 98 puntos se considera que sigue un modelo de enseñanza tradicional, los que obtienen una puntuación entre 98 y 147 están en transición hacia un modelo de enseñanza por competencias y los que obtienen una puntuación entre 147 y 196 son muy competentes al respecto.

Tabla 2. Clasificación del profesorado en función de su modelo de enseñanza.

\begin{tabular}{|c|c|c|}
\hline \multicolumn{2}{|c|}{ PUNTUACIÓN OBTENIDA EN EL CUESTIONARIO } \\
\hline 98 puntos & $98-147$ puntos & $147-196$ puntos \\
\hline Enseñanza tradicional & Modelo en transición & Trabajo por competencias \\
\hline
\end{tabular}

Fuente: Elaboración propia

Cabe decir que ningún docente se sitúa en un modelo de enseñanza tradicional, el $68,24 \%$ se sitúa entre 98 y 147 puntos lo que significa que están comenzando a trabajar por competencias con sus estudiantes, aunque todavía necesitan trabajar algunos aspectos. Sólo el 31,76\% del profesorado manifiesta que trabaja por competencias.

\section{Análisis del quehacer profesional de los docentes que trabajan por competencias}

Seguidamente se analizan las respuestas del profesorado que se encuentra dentro del modelo de trabajo por competencias, para lo que se abordará de forma individual 
cada uno de los ítems, que se agruparan en función de los diferentes elementos curriculares y de la organización de los centros.

Para comenzar, se recoge que, siempre o frecuentemente, el 88,9\% del profesorado trabaja todas las competencias desde su asignatura (ítem 15), el 81,5\% establece diferentes niveles de desempeño para el desarrollo progresivo de las competencias (ítem 30) y el $77,7 \%$ especifica diferentes aspectos de las competencias para cada curso (ítem 32). El $74,1 \%$ piensa que siempre ha de existir vinculación entre objetivos y competencias (ítem 18) y el 70,4\% siempre elabora los objetivos pensando en atender de modo flexible a las posibilidades de logro de cada estudiante (ítem 37). El desarrollo de la autonomía del alumnado es un objetivo para el profesorado (ítem 31), por lo que es incluido en su programación docente (el 63\% lo hace siempre y el 37\% frecuentemente).

En relación a los contenidos, en el ítem 3 el profesorado señala que considera prioritario que el alumnado sea capaz de relacionar los contenidos entre sí (el 70,4\% dice que deben hacerlo siempre y el 25,9\% que frecuentemente), aunque son menos (sólo el 11,1\% lo hace siempre) los que suelen plantearlos de modo disciplinar vinculándolos con otras áreas (ítem 7). A la hora de trabajar los contenidos, el profesorado nunca o pocas veces sigue estrictamente las indicaciones del libro, sin añadir nada (ítem 14), suele conectarlos con ejemplos de situaciones reales (lo hace siempre el 77,8\%) (ítem 38), frecuentemente parten de elementos de la vida cotidiana del alumnado (51,9\%) (ítem 2), y el 59,3\% del profesorado a veces se dedica principalmente en el aula a exponer los contenidos (ítem 27).

El profesorado al diseñar las actividades dirigidas al alumnado toma en consideración, siempre o frecuentemente, el desarrollo de las competencias (ítem 47), el contenido que se trabajará -81,5\% - (ítem 10); las situaciones que tendrán que afrontar sus estudiantes en su vida cotidiana (ítem 28); las situaciones de la vida real -81,5\% - (ítem 6); que admitan diferentes modos y niveles de resolución -92,6\% - (ítem 13); que sean interdisciplinares para desarrollar las competencias del alumnado -85,2\%\%(ítem 40); y que admitan niveles de dificultad y supongan un reto para el alumnado -92,5\%- (ítem 4). Además, en el diseño suelen tener en cuenta los contenidos de su asignatura $-40,7 \%$ frecuentemente y $48,1 \%$ a veces- (ítem 22), y en menor medida los de otras asignaturas $-11,1 \%$ siempre y $44,4 \%$ frecuentemente- (ítem 8 ). En cuanto a la resolución de dichas actividades, cabe indicar que nunca $(22,2 \%)$ o pocas veces (59,3\%) suele recogerse la solución en el libro de texto (ítem 16), para el 66,7\% a veces se encuentra de forma literal en la teoría dada (ítem 19), y, frecuentemente (63\%) requiere que los alumnos busquen y seleccionen libremente la información que necesitan (ítem 24). 
El $81,5 \%$ del profesorado utiliza en el aula, a veces o frecuentemente, materiales diseñados principalmente con una finalidad educativa, como libros de texto o software educativo (ítem 5). El 44,4\% del profesorado afirma que a veces utiliza el libro de texto para que los estudiantes accedan a los contenidos (ítem 23), siendo para el 66,6\% una fuente más a emplear (ítem 34). Además, el 96,3\% utiliza en sus clases habitualmente materiales de la vida cotidiana dándoles un uso didáctico (ítem 46), llegando el 92,6\% a utilizar materiales de elaboración propia en función de la actividad planteada (ítem 49).

La evaluación es planteada por el 85,1\% del profesorado como una buena ejecución de la práctica (ítem 39), por el 77,7\% como la comprobación de la articulación de conocimientos teóricos y prácticos (ítem 33) y sólo el 3,7\% del profesorado la plantea frecuentemente como la reproducción de contenidos teóricos (ítem 9). El 55,6\% del profesorado siempre utiliza los criterios de evaluación y los estándares de aprendizaje para evaluar tanto los objetivos como el grado de desempeño de las competencias (ítem 43). La autoevaluación y/o la coevaluación son utilizadas siempre por el 18,5\% del profesorado para favorecer la motivación (ítem 20), mientras que el 25,9\% las usa para facilitar tanto el aprendizaje significativo como la reflexión sobre el propio aprendizaje (ítem 48). En lo relativo a las sesiones de evaluación, parece que el 44,4\% del profesorado reconoce que se centran tanto el desarrollo de las competencias clave de cada estudiante como los resultados en cada una de las asignaturas (ítem 36), sólo el 29,6\% del profesorado afirma que se discute siempre sobre el grado en que los estudiantes han alcanzado las distintas competencias (ítem 21), mientras que el 74\% reconoce que pocas veces o nunca se centran sólo en las notas obtenidas por cada estudiante en los exámenes y prácticas (ítem 44).

Trabajar por competencias exige de un clima colaborativo en los centros educativos. De ahí, que el $74,1 \%$ del profesorado afirme trabajar frecuentemente o siempre en grupos interdisciplinares (ítem 1) y el 66,6\% lo haga habitualmente con el pequeño grupo de profesores con el que comparte asignatura o área de conocimiento (ítem 17). Cabe decir, que el $37 \%$ del profesorado que se sitúa en el modelo de trabajo por competencias pertenece al mismo centro.

Finalmente, en el ítem 45, cuando se les pregunta por su participación en cursos de formación docente para el desarrollo de competencias, reconocen que es baja. De ahí que afirmen, en el ítem 42, que en los últimos años han echado en falta cursos de formación en los que se trabajase el desarrollo de competencias y su aplicación en el aula.

La formación del profesorado podría dirigirse a fortalecer aquellos aspectos susceptibles de mejora, detectados mediante el cuestionario porque su valor medio es inferior a 3 (indica que se realiza puntualmente), lo que supone no realizar dicha acción "frecuentemente" (3) o "siempre" (4), y recogidos en el Gráfico 1. 


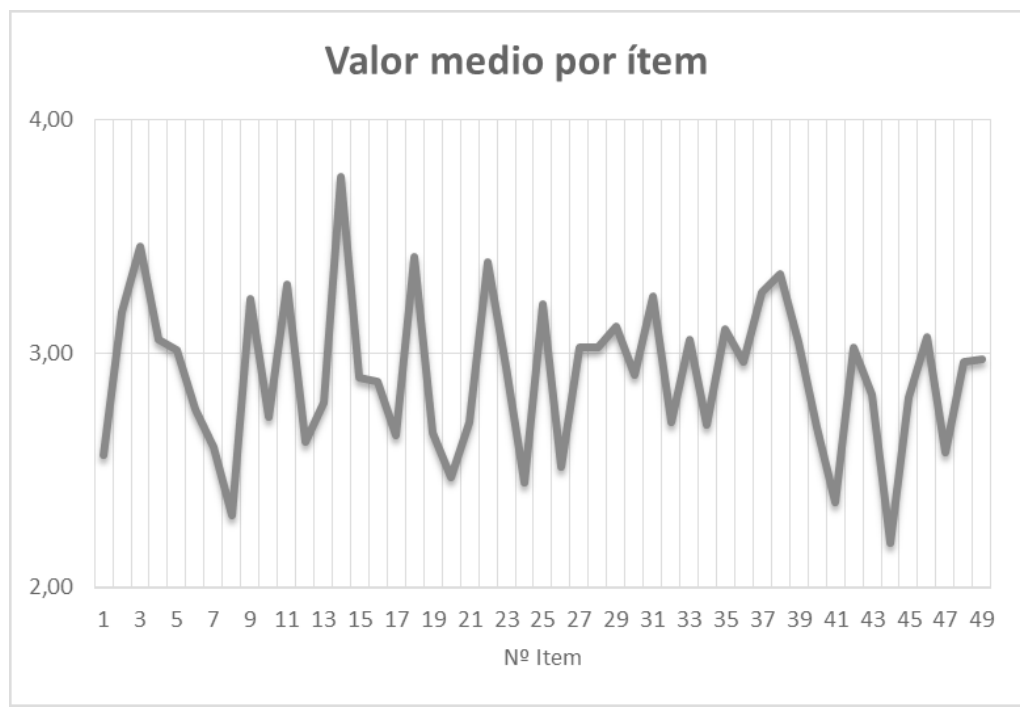

Gráfico 1. Valoración media de los ítems del cuestionario

\section{Fuente: Elaboración propia}

Teniendo en cuenta la valoración de los ítems, recogida en la tabla 1, se podrían indicar dos aspectos clave en la formación y que a su vez son fundamentales a la hora de plantear el trabajo por competencias. El primero sería el trabajo en equipo, con sus implicaciones a la hora de trabajar contenidos y diseñar actividades de modo interdisciplinar vinculándolos a otras áreas, o a la hora de valorar su grado de consecución. El segundo señala hacia la evaluación de competencias, con el uso de criterios de evaluación y estándares de aprendizaje y la utilización de técnicas de recogida de información variadas y que impliquen la reflexión del alumnado sobre su propio aprendizaje.

\section{Discusión y conclusiones}

El perfil docente de un profesor que trabaja por competencias y que hemos descrito a nivel teórico se corresponde con el que se establece cuando se analiza cada uno de los ítems, y aunque todavía queda por conseguir que algunas acciones pasen de desarrollarse frecuentemente a siempre, son muchos los puntos fuertes del profesorado que está trabajando en la línea marcada por las administraciones educativas. Cabe destacar el trabajo en grupo como punto fuerte, dado que está considerado como una de las herramientas más poderosas para la consecución de objetivos en cualquier ámbito, sector o actividad. Y como no podía ser menos, el trabajo por competencias 
también lo precisa, aunque todavía sea preciso fomentar la relación entre contenidos de diferentes áreas, por ejemplo.

Tal y como han demostrado los resultados analizados, es posible afirmar que la Educación de Adultos en Asturias ha abandonado el modelo tradicional basado en la transmisión de contenidos en el que el alumno adopta un papel pasivo ante su propio aprendizaje.

Aunque a tenor de lo planteado en esta investigación parezca que la evaluación de las competencias acaba siendo una evaluación sobre las actividades y la metodología utilizada por los docentes, sí es cierto que, a diferencia de la enseñanza centrada en los contenidos, sin un buen planteamiento metodológico el avance no es viable, ya que el desarrollo y adquisición de las competencias esperadas depende fundamentalmente de las oportunidades o situaciones en las que los estudiantes tengan que desenvolverse. El rol del docente se encuentra en proceso de transformación, aún algunos profesores consideran que enseñar es principalmente transmitir información, pero se está caminando hacia el rol del docente que genera y gestiona espacios de aprendizaje, que guía a sus alumnos en el proceso personal de autogestión de su aprendizaje del que es el verdadero protagonista. No obstante, para conseguir este cambio es preciso que se realice una formación específica dirigida al profesorado, tal y como ellos demandan.

\section{Agradecimientos}

Este trabajo ha sido financiado por la Comisión Europea (acción estratégica KA2) en el marco del Proyecto: Sharing effective educational practices and systematizing a training competences programme for employment and inclusion for vulnerable adults (Ref: 2016-1-ES01-KA204-025159).

\section{Referencias}

Bolarín Martínez, M.J., Moreno Yus, M.A., Porto Currás, M. (2013). Coordinación docente e interdisciplinariedad: análisis de su contribución a la adquisición de competencias docentes y discentes. Revista de Docencia Universitaria, 11(2), 443-462.

Cárdenas-Rodríguez, R., Terrón-Caro, T. Monreal-Gimeno, C. (2015). Interdisciplinariedad o multidisciplinariedad en el ámbito universitario. Desafíos para la coordinación docente. Bordón, 67(3), 167-183. DOI: 10.13042/Bordon.2015.67309.

Ferreira, F., Henriques-Coelho, T. (2016). Aprendizagem baseada na resolução de problemas: impacto no desenvolvimento do pensamento crítico. Revista Lusófona de Educação, 32, 123-137 [Disponível em https://revistas.ulusofona.pt/index.php/rleducacao/article/view/5519, consultado em 7 de Agosto de 2019].

Hidalgo, J., de la Blanca, S., Risueño, J.J. (2011). La innovación educativa en la educación de adultos como mejora del proceso de enseñanza-aprendizaje. Escuela Abierta, 14, 83-109.

Karrera, I., Zulaika, T., Aldaz, J. (2014). Actuaciones interdisciplinares del profesorado universitario: desarrollo y evaluación de las competencias genéricas de Grado en la formación de estudiantes de Educación Primaria. Profesorado. Revista de Curriculum y Formación del profesorado, 18(2), 285-303. 
Lavega, P., Sáez De Orcáriz, U., Lasierra, G., Salas, C. (2013). Intradisciplinariedad e Interdisciplinariedad en la adquisición de competencias: estudio de una experiencia de aprendizaje cooperativo. Revista Electrónica Interuniversitaria de Formación del Profesorado, 16(1), 133-145. DOI: http://dx.doi. org/10.6018/reifop.16.1.179491.

Mateo J., Vlachopoulos, D. (2013). Reflexiones en torno al aprendizaje y a la evaluación en la universidad en el contexto de un nuevo paradigma para la educación superior. Educación XX1, 16(2), 183-208. DOI: 10.5944/educxx1.16.2.2639.

Medina Fernández, O. (2000). Especificidad de la educación de adultos. Bases psicopedagógicas y señas de identidad. Educación XX1, 3, 91-140.

Medina Fernández, O., Sanz Fernández, F. (2009). El reconocimiento y la acreditación de la experiencia. Teoría de la Educación, 21(2), 165-193.

Méndez-Giménez, A., Sierra-Arizmendiarrieta, B., Mañana-Rodríguez, J. (2013). Percepciones y creencias de los docentes de Primaria del Principado de Asturias sobre las competencias básicas. Revista de Educación, 362, 737-761. DOI: 10.4438/1988-592X-RE-2013-362-248.

Ministerio de Educación y Formación Profesional (2018). Enseñanzas no universitarias. Estadística del profesorado y otro personal. Curso 2017-2018. Datos avance. http://www.mecd.gob.es/serviciosal-ciudadano-mecd/estadisticas/educacion/no-universitaria/profesorado/estadistica/2017-2018-DA. html.

Monarca, H. y Rappoport, S. (2013). Investigación sobre los procesos de cambio educativo: el caso de las competencias básicas en España. Revista de Educación, Extraordinario, 54-78. DOI: 10.4438/1988-592X-RE-2013-EXT-256.

Palomares Montero, D., Chisvert-Tarazona, M.J. (2016). El aprendizaje cooperativo: una innovación metodológica en la formación del profesorado, Cultura y Educación, 28(2), 378-395, DOI: 10.1080/11356405.2016.1158448.

Poblete, M., Villa, A. (2011). SEBSCO, una experiencia alternativa para evaluar competencias. Aula Abierta, 39(3), 15-30.

Recomendación del Parlamento Europeo y del Consejo, 2006/962/CE, de 18 de diciembre de 2006 sobre las competencias clave para el aprendizaje permanente.

Rubio Hurtado, M.J., Ruiz Bueno, A., Martínez-Olmo, F. (2016). Percepción del alumnado sobre la utilidad de las actividades de aprendizaje para desarrollar competencias. Revista de Investigación Educativa, 34(1), 221-240. DOI: http://dx.doi.org/10.6018/rie.34.1.225131.

Sáiz Serrano, J. (2011). Actividades de libros de texto de Historia, competencias básicas y destrezas cognitivas, una difícil relación: análisis de manuales de $1^{\circ}$ y $2^{\circ}$ de ESO. Didáctica de las Ciencias Experimentales y Sociales, 25, 37-64.

Sánchez Mirón, B. y Boronat Mundina, J. (2014). Coaching educativo: modelo para el desarrollo de competencias intra e interpersonales. Educación XX1, 17(1), 221-242. DOI: 10.5944/ educxx1.17.1.1072.

Sarrate Capdevila, M.L, Pérez de Guzmán Puya M.V. (2005). Educación de personas adultas. Situación actual y propuestas de futuro. Revista de Educación, 336, 41-57.

Sierra-Arizmendiarrieta, B., Méndez-Giménez, A., Mañana-Rodríguez, J. (2013). La programación por competencias básicas: hacia un cambio metodológico interdisciplinar. Revista Complutense de Educación, 24(1), 165-184. 
Sierra-Arizmendiarrieta, B., Pérez-Ferra, M. (2015). La educación en J.J. Rousseau: un antecedente metodológico de la enseñanza basada en la formación de competencias, Revista Complutense de Educación, 26(1), 121-139.

Silva, H., Lopes, J., Dominguez, C., Payan-Carreira, R., Morais, E., Nascimento, M., Morais, F. (2016). Fostering critical thinking through peer review between cooperative learning groups. Revista Lusófona de Educação, 32, 31-45.

Villa, A., Poblete, M. (2007). Aprendizaje Basado en Competencias. Una propuesta para la evaluación de las competencias genéricas. Bilbao: Mensajero.

Villa Sánchez, A., Poblete Ruiz, M. (2011). Evaluación de competencias genéricas: principios, oportunidades y limitaciones. Bordón, 63(1), 147-170.

Villardón Gallego, L. (2006). Evaluación del aprendizaje para promover el desarrollo de competencias. Educatio Siglo XXI, 24, 57-76.

Susana Molina Martín

Grupo de investigación ZINTAC Facultad de Formación del Profesorado y Educación -

Universidad de Oviedo

Email: smmolina@uniovi.es

ORCID: https://orcid.org/0000-0001-9467-904X

\section{Beatriz Sierra-Arizmendiarrieta}

Grupo de investigación ZINTAC

Facultad de Formación del Profesorado y Educación -

Universidad de Oviedo

Email: bsierra@uniovi.es

ORCID: https://orcid.org/0000-0002-9002-3737

Marta Soledad García Rodríguez

Grupo de investigación ZINTAC

Facultad de Formación del Profesorado y Educación -

Universidad de Oviedo

Email: martagar@uniovi.es

ORCID: https://orcid.org/0000-0003-1682-6088

Correspondência

Susana Molina Martín

Facultad de Formación del profesorado y Educación. C/Aniceto Sela s/n, 33005-Oviedo

Data de submissão: Novembro 2018

Data de avaliação: Janeiro 2019

Data de publicação: Setembro 2019 